\title{
Outflow activity in brown dwarfs
}

\section{Emma T. Whelan ${ }^{1}$, Thomas P. Ray ${ }^{1}$, Francesca Bacciotti ${ }^{2}$, Sofia Randich ${ }^{2}$, Ray Jayawardhana ${ }^{3}$, Antonella Natta ${ }^{2}$, Leonardo Testi ${ }^{2}$ and Subu Mohanty ${ }^{4}$}

\author{
${ }^{1}$ Dublin Institute for Advanced Studies \\ email: ewhelan@cp.dias.ie \\ ${ }^{2}$ Osservatorio Astrofisico di Arcetri \\ ${ }^{3}$ Department of Astronomy and Astrophysics, University of Toronto \\ ${ }^{4}$ Harvard-Smithsonian Center for Astrophysics
}

\begin{abstract}
While numerous studies have been aimed at understanding the properties of young brown dwarfs relatively little exploration of their potential as drivers of outflows has occurred. Forbidden emission lines are important probes of outflows from young stellar objects, as they trace the shocks which form as an outflow interacts with the ambient medium of its driving source. While forbidden emission was identified in the spectra of young brown dwarfs, indicating the presence of outflows, these lines were weak and confined to the brown dwarf continuum position. Hence their origin in an outflow could not be confirmed. Our approach to this problem, is to analyse the forbidden line regions of brown dwarfs using spectro-astrometry. Spectroastrometry is a novel technique which allows the user to recover spatial information from a spectrum beyond the limitations of the seeing of the observation. Using this technique we have found two brown dwarf outflows to date. In this chapter we outline this technique, describe our results for the brown dwarfs $\rho$-Oph 102 and 2MASS1207-3932 and discuss our future plans.
\end{abstract}

Keywords. 2MASSWJ1207334-393254, stars: low mass, brown dwarfs, stars: formation, ISM: jets and outflows.

\section{Introduction}

The study of young brown dwarfs (BDs) in star forming regions has a special importance as clearly the first question that must be asked about these objects is how are they formed? More and more BDs are being discovered and analysed in nearby stellar nurseries such as Taurus (see Briceño et al. 1998; Luhman et al. 2003; Muzerolle et al. 2005; Grosso et al. 2007), Ophiuchius (Wilking et al. 1999; Allers et al. 2007) or Orion (Lucas \& Roche 2000). It is a reasonable first step towards understanding BD formation to compare them with low mass YSOs, in particular the classical T Tauri stars (CTTSs). Specific questions are, do BDs undergo accretion and have accretion disks? and if they are accreting material do they drive outflows?

Many studies have addressed the question on the similarity between young BDs and CTTSs and it is now apparent that BDs and CTTSs bear a strong resemblance. The presence of accretion disks has been confirmed from a study of the spectral energy distributions (SEDs) of a number of BD candidates (Natta et al. 2002; Jayawardhana et al. 2003a) and Jayawardhana et al. (2003b) demonstrated that high resolution optical spectra point to the existence of a T Tauri like accretion phase for BDs. While BDs have been shown to be active accretors (Scholz \& Jayawardhana 2006) relatively little is known about their outflow activity. As outflows in CTTSs are directly related to magnetospheric infall (Hartigan et al. 1994; Königl \& Pudritz 2000), it is feasible that BDs demonstrating strong accretion will drive outflows. 


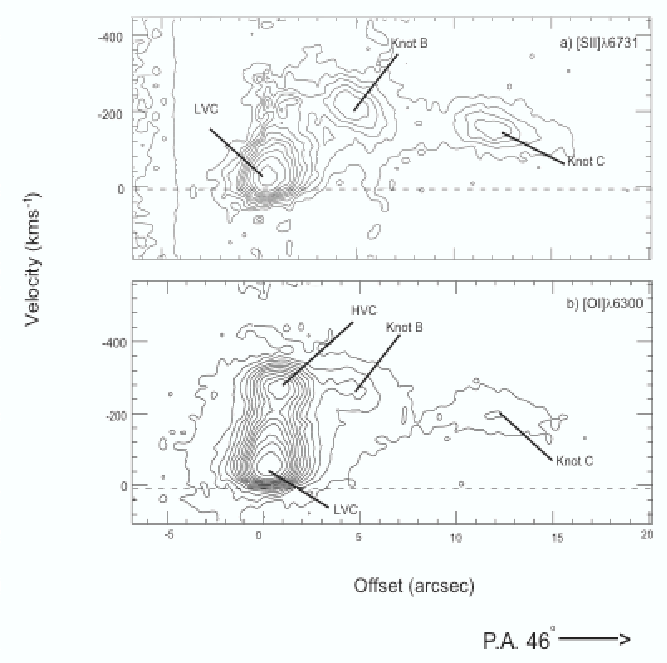

Figure 1. Continuum-subtracted position velocity diagrams of the optical forbidden emission lines from DG Tau taken from Whelan et al. (2004). This Figure illustrates the importance of the FELs as tracers of outflows from CTTSs. Note the comparison between the DG Tau jet and the PV diagram of the [OI] $\lambda 6300$ emission from the BD 2MASS1207-3932 presented in Figure 5 .

Early indications that BDs drove outflows came from the discovery of forbidden emission lines (FELs) e.g. [OI] $\lambda \lambda 6300,6363$ in their optical spectra (Fernández \& Comerón 2001; Muzerolle et al. 2003). FELs are important coolants of interstellar shocks and thus are strong tracers of outflow activity (see Figure 1). In the case of BDs however, the FEL regions were weak and not extended. Hence their formation in an outflow while likely could not at first be confirmed. Using the novel technique of spectro-astrometry we have achieved this confirmation and have thus far discovered two BD outflows. Below we shall describe in detail the spectro-astrometric technique and the results we have achieved thus far in the study of young BDs. In the final section we shall outline our future plans.

The discovery that BDs have outflows while relevant to our overall understanding of BD formation is perhaps primarily relevant to the study of the outflow phenomenon. That BDs can launch outflows emphasises the robustness of the outflow mechanism over an enormous range of masses (up to $10^{8-9} \mathrm{M}_{\odot}$ in Active Galactic Nuclei). Indeed the second BD found to have an outflow, 2MASS1207-3932, is only a $24 \mathrm{M}_{J U P}$ object thus it is now the lowest mass galactic object that is actively driving an outflow.

\section{Spectro-astrometric analysis of the BD FELs}

As stated the FELs found in the spectra of young BDs were weak and confined to the source position. Our aim was to recover the position of the emission regions with respect to the $\mathrm{BD}$ position using spectro-astrometry. In order to spectrally resolve the BD FEL regions with a usuable $\mathrm{S} / \mathrm{N}$, observations were made using the UV-Visual Echelle Spectrometer (UVES) on the VLT. High resolution $(\mathrm{R}=40,000)$ cross-dispersed spectra (CD3 disperser, spectral range 4810-6740 ^) were obtained on the ESO VLT UT2.

Spectro-astrometry simply translates as a measurement of the position of the centroid of a flux distribution, as a function of wavelength (hence velocity for a line), producing a plot of offset versus velocity. The profile of a star is smeared by atmospheric turbulence 


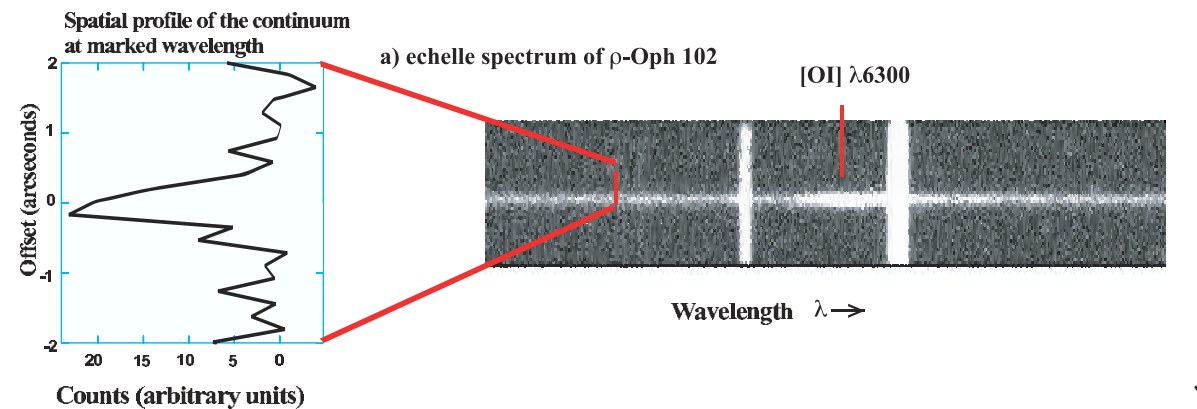

Counts (arbitrary units) $\quad$ Spatial profile of the $[\mathrm{OI}] \lambda 6300$ emission line region at marked wavelength

b) Continuum subtracted spectrum

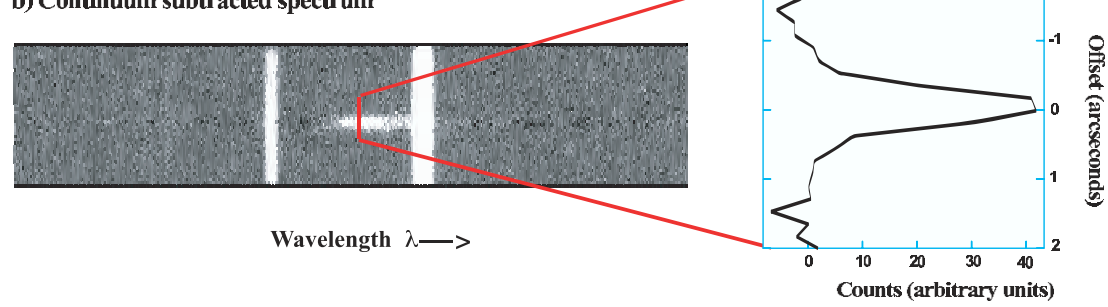

Figure 2. The principles underlying the technique of spectro-astrometry illustrated for the brown dwarf $\rho$-Oph 102. In the FEL spectrum of a young brown dwarf (pure emission line region originating in the outflow plus $\mathrm{BD}$ continuum emission) the contribution from the continuum can be removed by interpolation across the line. The emission centroid of the FEL will be shifted from the continuum centroid (forbidden line emission is quenched close to the star where densities far exceed the critical density.) This shift is accuately measured through Gausian fitting of the spatial profile and through exploring the shifts/offsets with respect to the continuum, one can recover spatio-kinematic information about the flow at milliarcsecond scales.

to appear Gaussian (at least to a first approximation) rather than point-like. Hence, the centroid of the emission is measured by fitting a Gaussian to the spatial profile extracted from the 2D spectrum, at each wavelength or pixel (see Figure 2). If an emission line e.g. a FEL is actually formed in an outflow, positional shifts (weighted by the line intensity) with respect to the continuum centroid are measured in the direction of the outflow (see Whelan et al. 2004). Figure 2 illustrates the process of applying spectro-astrometry to $[\mathrm{OI}] \lambda 6300$ region in the spectrum of $\rho$-Oph 102 . Note that before the position of the pure line emission can be mapped the continuum must be removed. Contamination by the continuum emission will tend to drag the line position back towards the source.

While the width of the spatial profile is determined by the seeing, how accurately one can determine the centroid of the emission is, in theory (for fixed seeing), limited only by the strength of the observed signal to noise ratio. The spectro-astrometric accuracy is given by $\sigma_{\text {centroid }}=$ Seeing $/ 2.355 \sqrt{N_{p}}$ where $\mathrm{N}_{p}$ is the number of detected photons (see Bailey et al. 1998). Hence increasing the total number of detected photons increases the astrometric accuracy so that milli-arcsecond precision is possible. Although the BD forbidden emission was relatively very weak (compared to the average CTTS say) the $\mathrm{BD}$ continuumm emission was also weaker or comparable to the forbidden emission. This is evident from Figure 2. In order to properly compare the line and continuum our approach was to bin the line and continuum in such a way that the accuracy to which we measured the position of each was the same. Lastly an important consideration when using spectro-astrometry is the possibility of spectro-astrometric artifacts (see Brannigan et al. 2006). In our analysis lines like HeI $\lambda 6678$ and LiI 6708 were found to have 


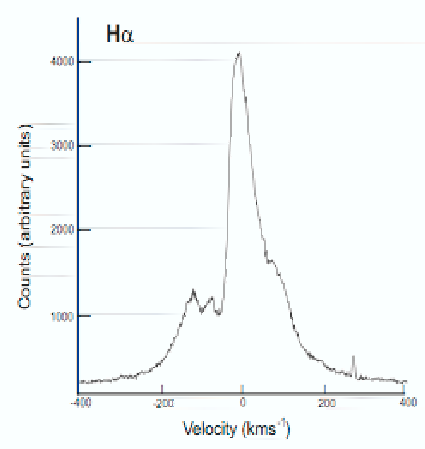

Figure 3. The outflow signature in the $\mathrm{H} \alpha$ profile of $\rho$-Oph102. Again this is taken from Whelan et al. (2005). The P Cygni-like dip in the line profile is a strong signature of outflow activity. $\mathrm{H} \alpha$ emission from the brown dwarf is absorbed as it passes through material moving outwards along our line of sight. Because this material is moving towards us, the dip is on the blueward side of the line. CTTSs are strong $\mathrm{H} \alpha$ emitters, and $\mathrm{P}$ Cygni $\mathrm{H} \alpha$ profiles originally confirmed that such protostars drive outflows. The dip in the $\mathrm{H} \alpha$ emission of $\rho$-Oph102 is at approximately the outflow radial velocity determined from the forbidden lines.

no spectro-astrometric signal. As these lines are chromospheric and photospheric respectively no offset is expected. The lack of positional displacement in such lines rules out the possibility of spectro-astrometric artifacts.

\section{Rho Oph 102}

The $\sim 60 \mathrm{M}_{J U P} \mathrm{BD} \rho$-Oph 102 was the first BD to be confirmed to have an outflow and these first exciting results were presented in Whelan et al. (2005). The UVES spectra of $\rho$-Oph 102 obtained in May 2003 revealed the [OI] $\lambda \lambda 6300,6363$, [NII] $\lambda 6583$ and [SII] $\lambda \lambda 6716,6731$ FELs to be present (Natta et al. 2004). Our analysis put the average velocity of the FELs at $\sim-45 \mathrm{kms}^{-1}$ suggesting mass outflow. Moreover the blue-shifted asymmetry mirrored what is seen in CTTSs, and pointed to the presence of a disk. A second indication that an outflow was present came from the $\mathrm{H} \alpha$ line (see Figure 3). Its profile is clearly asymmetrical, i.e., the blue-shifted wing of the line appears to be absorbed in a P-Cygni like fashion at a similar velocity to the FELs $\left(\sim-80 \mathrm{kms}^{-1}\right)$. However a classical P-Cygni profile, i.e., one that dips below the continuum, is not observed but such a profile is, in any event, a rare occurrence even amongst the CTTSs.

Of the five FELs detected only the $[\mathrm{OI}] \lambda \lambda 6300,6363$ and $[\mathrm{SII}] \lambda 6731$ lines were strong enough for any spectro-astrometric analysis. This analysis is presented in Figure 4. Overall the centroids of all the FELs are displaced to the south, i.e. have negative offsets with respect to the continuum and these offsets reach a maximum of 0."08-0." 1 at a blueshifted velocity of $\sim-41 \mathrm{kms}^{-1}$. The scale of this blue-shifted offset would suggest a minimum (projected) disk radius of $0 . " 1(\geqslant 15$ AU at the distance of the $\rho$-Ophiuchi cloud) in order to hide any red-shifted component. There is no clear spatial offset in $\mathrm{H} \alpha$ even though its higher signal to noise potentially would allow one to measure even smaller offsets than observed in the forbidden lines. This is in agreement with the idea that most of the $\mathrm{H} \alpha$ emission arises from accretion on much smaller scales than are being probed here (see Natta et al. 2004). Lastly, no offset is measured in the Li $\lambda 6708$ and HeI $\lambda 6678$ lines. As explained above this lack of positional displacement rules out the existence of spectro-astrometric artifacts. 

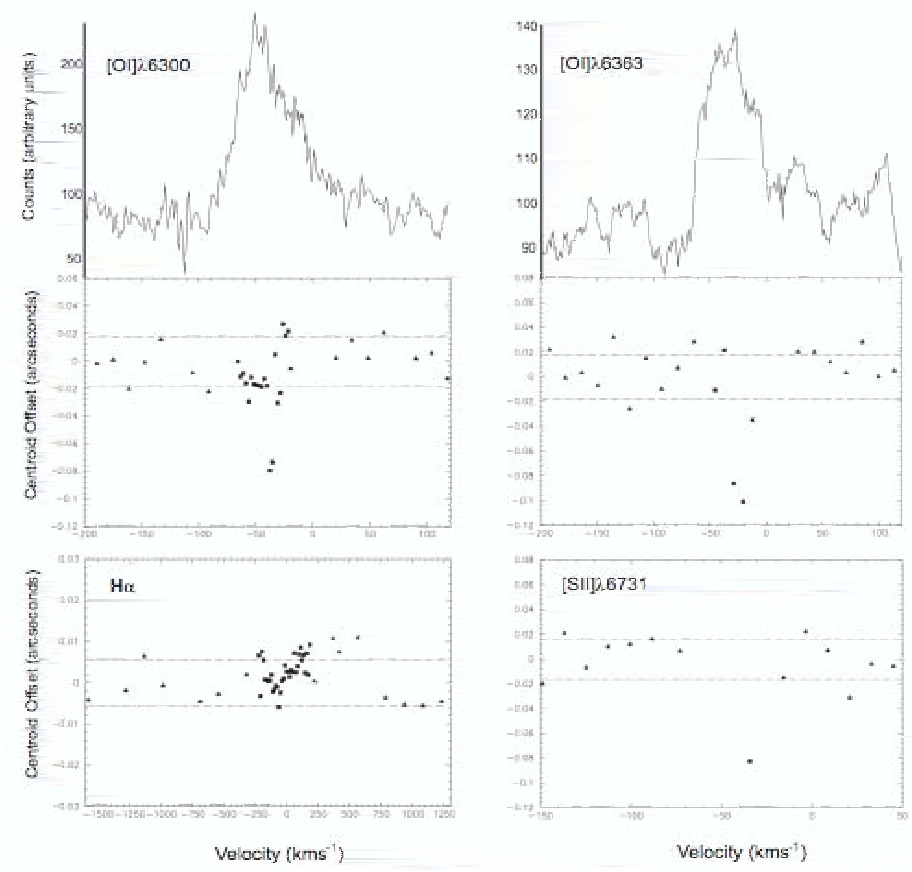

Figure 4. Line profiles (top row) and spectro-astrometric plots (middle row) for the $[\mathrm{OI}] \lambda 6300$ and $[\mathrm{OI}] \lambda 6363$ doublet and spectro-astrometric plots (bottom row) for the $\mathrm{H} \alpha$ and [SII] $\lambda 6731$ lines. These results were published in Whelan et al. (2005). Continuum and line offset points are represented by black triangles and squares respectively. All velocities are systemic and spatial offsets are in the north-south direction (in arcseconds) with negative offsets to the south. Dashed lines delineate the $\pm 1 \sigma$ error envelope. For $\mathrm{H} \alpha$, note the much smaller offset scale. The [SII] line is blue-shifted to around $-40 \mathrm{kms}^{-1}$.

\section{2MASS1207}

Following our recent discovery of a bipolar outflow from the $\sim 24 \mathrm{M}_{J U}$ BD $2 \mathrm{MASS} 1207-$ 3932 , this BD is now the lowest mass galactic object known to drive an outflow (see Whelan et al. 2007). The nature of this BD as a strong accretor was clearly evident from early observations. Mohanty et al. (2003) reported bright asymmetric $\mathrm{H} \alpha$ emission with a full width at $10 \%$ of the peak flux, of $>200 \mathrm{kms}^{-1}$. Also Mohanty et al. (2005) were the first to observe the $[\mathrm{OI}] \lambda 6300$ line in the spectrum of 2MASS1207 suggesting it could be driving an outflow. Our UVES spectra were obtained in May 2006 and the $[\mathrm{OI}] \lambda \lambda 6300$, 6363 lines were the only "traditional" FEL tracers seen in the spectrum. A position velocity $(\mathrm{PV})$ diagram of the smoothed $[\mathrm{OI}] \lambda 6300$ spectrum is presented in Figure 5. For this BD the 2D UVES spectra were smoothed using an elliptical Gaussian filter of FWHM $0.12 \AA \times 0 . " 22$. The Gaussian smoothing improved the signal to noise and effectively decreased the spectral and spatial resolution to $\mathrm{R} \sim 8000$ and $0 . " 7$ respectively. The PV diagram shows blue and red-shifted emission at velocities of $\sim-8 \mathrm{kms}^{-1}$ and $\sim+4 \mathrm{kms}^{-1}$ and a relative displacement between the two parts of the line is apparent. This displacement is recovered using spectro-astrometry. Note that the relatively very small radial velocity of the outflow is consistent with the near edge-on disk hypothesis for 2MASS1207-3932.

The results of a spectro-astrometric analysis of the smoothed spectrum are shown in Figure 6. Here the continuum and $[\mathrm{OI}] \lambda 6300$ line emission were smoothed so that the spectro-astrometric error in both regions are the same. The blue and red-shifted 


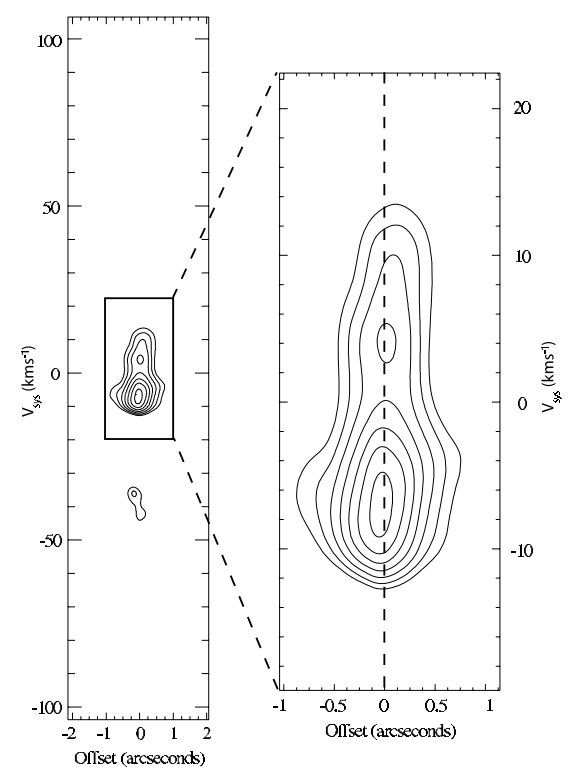

Figure 5. Continuum subtracted Position Velocity diagram of the $[\mathrm{OI}] \lambda 6300$ line in 2MASS1207-3932. Here the spectrum was smoothed in both the spectral and spatial directions using an elliptical Gaussian function. The contours begin at 3 times the r.m.s noise and increase in intervals of the r.m.s noise. The red and blue-shifted components to the line are obvious and an opposing offset in these components is suggested. The offset is recovered using spectro-astrometry.

components to the $[\mathrm{OI}] \lambda 6300$ line are found to be offset in opposing directions to an absolute distance of $\sim 80$ mas (see Figure 6 ). Hence it is clear that the $[\mathrm{OI}] \lambda 6300$ emission originates in a faint bipolar outflow driven by 2MASS1207. The 1- $\sigma$ error in the measurements of 18 mas, is marked by the dashed line in Figure 6. Other lines of interest are $[\mathrm{OI}] \lambda 6363, \mathrm{H} \alpha$ and $\mathrm{HeI} \lambda 6678$. The $[\mathrm{OI}] \lambda 6363$ is $\sim 3$ times fainter than the $[\mathrm{OI}] \lambda 6300$ line and hence is just below the detection limit in the raw spectrum. By smoothing the spectrum using a large gaussian filter (FWHM in dispersion direction $=0.35 \AA$ ) the $[\mathrm{OI}] \lambda 6363$ is revealed at a similar velocity to the $[\mathrm{OI}] \lambda 6300$ line, although detailed kinematic data is lost. The primary origin of the $\mathrm{H} \alpha$ and $\mathrm{HeI} \lambda 6678$ lines is in the accretion flow. As this occurs on a very small scale we expect to measure no spectro-astrometric offset and, as can be seen in Figure 6 none is found, again ruling out the presence of spectro-astrometric artifacts.

\section{Future Work}

While it is generally accepted that accretion activity in BDs is simply scaled down from infall in CTTSs (Jayawardhana et al. 2003b), the presumption that BDs also drive scaled-down versions of $\mathrm{T}$ Tauri jets and outflows while credible has yet to be generally confirmed. The evidence gathered so far supporting the continuation of the CTT paradigm for outflow activity, into the young BD mass regime, includes the blue-shifted nature of the FELs (mimicking what is seen in the majority of CTTSs) and that the measured offsets lie withing the range estimated for a BD (Whelan et al. 2005, 2007 for further details). We plan to develop the work described in this chapter by firstly increasing the sample of BDs known to drive outflows and secondly by working towards capturing the first images of BD outflows. Observing time has been granted on the VLT 

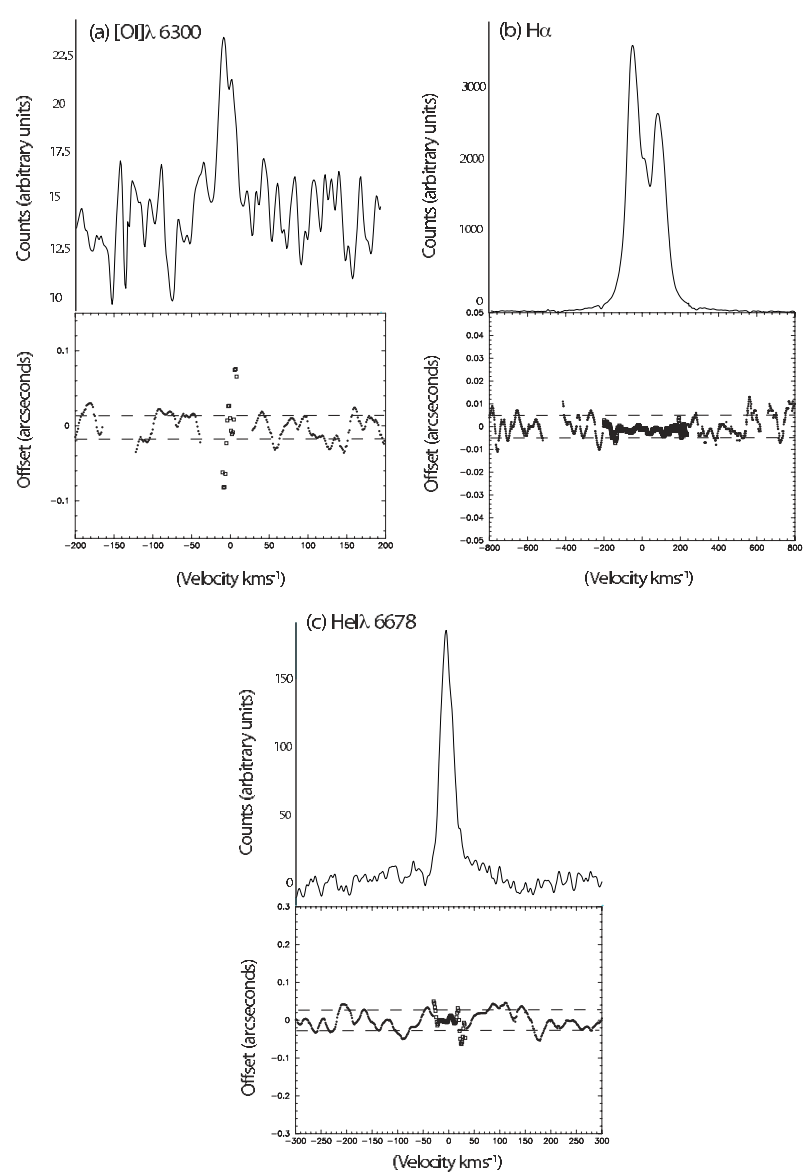

Figure 6. Offset velocity diagrams in the vicinity of the $[\mathrm{OI}] \lambda 6300, \mathrm{H} \alpha$ and $\mathrm{He} \mathrm{I}$ lines. The green dashed lines delineate the $\pm 1 \sigma$ error envelope for the centroid position of the continuum. The bipolar offset in the [OI] $\lambda 6300$ reveals the presence of an outflow. No offsets are measured in the $\mathrm{H} \alpha$ and $\mathrm{He}$ I lines as expected ruling out the possibility of spectro-astrometric artifacts.

to observe the optical spectra of four more BDs (known to be active accretors) and to image the outflows from $\rho$-Oph 102 and 2MASS1207-3932. Both these studies will allow us to further investigate how BD outflows compare to outflows driven by CTTSs.

In particular, questions that can be answered from images of the outflows from $\rho$-Oph 102 and 2MASS1207-3932 are relevant to the collimation, opening angles and morphology of BD outflows. Are BD outflows comparable to those of CTT jets or are they more or less collimated? Are BD outflows knotty? Current models of jet launching and collimation describe how infalling material from the surrounding envelope is re-launched out along the magnetic field lines (from the disk/star) and then collimated by magnetic hoop stresses. A measurement of the degree of collimation in the BD outflows will answer the question as to whether with spectro-astrometry we are detecting a jet or a much wider outflow and also address the role of magnetic fields in the formation of BD outflows. Indeed the fact that we see a bipolar outflow in 2MASS1207 already suggests that we are not detecting a spherically symmetric outflow but one that is axisymmetric. In addition models of jet launching link outflow directly to infall and direct observational evidence exists to support a strong correlation between infall in CTTSs and outflow activity (Hartigan et al. 1994). Each Herbig-Haro knot is related directly to an sudden burst in accretion activity hence 
jets are an excellent fossil record of the accretion history of the driving source. Episodic emission from the BDs in question (i.e knotty jets) would suggest episodic accretion and a similar origin to CTT jets. We would also have a record of the accretion history of the BD.

\section{References}

Allers, K. N., et al. 2007, The Astrophysical Journal, 657, 511

Bailey, J. A. 1998, SPIE-The International Society for Optical Engineering, 3355, 932

Briceño, C., Hartmann, L., Stauffer, J., \& Martín, E. 1998, The Astronomical Journal, 115, 2074

Brannigan, E., Takami, M., Chrysostomou, A., \& Bailey, J. 2006, Monthly Notices of the Royal Astronomical Society, 367, 315

Fernández, M., \& Comerón, F. 2001,Astronomy and Astrophysics, 380, 264

Grosso, N., et al. 2007, Astronomy and Astrophysics, 468, 391

Hartigan, P., Morse, J. A., \& Raymond, J. 1994, The Astrophysical Journal , 436, 125

Jayawardhana, R., Ardila, D. R., Stelzer, B., \& Haisch, K. E., Jr. 2003a, The Astronomical Journal, 126, 1515

Jayawardhana, R., Mohanty, S., \& Basri, G. 2003b, The Astrophysical Journal, 592, 282

Königl, A., \& Pudritz, R. E. 2000, Protostars and Planets IV, 759

Lucas, P. W. \& Roche, P. F. 2000,Monthly Notices of the Royal Astronomical Society, 314, 858

Luhman, K. L., Briceño, C., Stauffer, J. R., Hartmann, L., Barrado y Navascués, D., \& Caldwell, 2003, The Astrophysical Journal, 590, 348

Mohanty, S., Jayawardhana, R., \& Barrado y Navascués, D. 2003, The Astrophysical Journal Letters, 593, L109

Mohanty, S., Jayawardhana, R., \& Basri, G. 2005, The Astrophysical Journal, 626, 498

Muzerolle, J., Hillenbrand, L., Calvet, N., Briceño, C., \& Hartmann, L. 2003, The Astrophysical Journal, 592, 266

Muzerolle, J., Luhman, K. L., Briceño, C., Hartmann, L., \& Calvet, N. 2005, The Astrophysical Journal, 625, 906

Natta, A., Testi, L., Comerón, F., Oliva, E., D’Antona, F., Baffa, C., Comoretto, G., \& Gennari, S. 2002,Astronomy and Astrophysics, 393, 597

Natta, A., Testi, L., Muzerolle, J., Randich, S., Comerón, F., \& Persi, P. 2004,Astronomy and Astrophysics, 424, 603

Scholz, A., \& Jayawardhana, R. 2006, The Astrophysical Journal, 638, 1056

Whelan, E. T., Ray, T. P., \& Davis, C. J. 2004,Astronomy and Astrophysics, 417, 247

Whelan, E. T., Ray, T. P., Bacciotti, F., Natta, A., Testi, L., \& Randich, S. 2005, Nature, 435, 652

Whelan, E. T., Ray, T. P., Randich, S., Bacciotti, F., Jayawardhana, R., Testi, L., Natta, A., \& Mohanty, S. 2007, The Astrophysical Journal Letters, 659, L45

Wilking, B. A., Greene, T. P., \& Meyer, M. R. 1999, The Astronomical Journal, 117, 469 